\title{
Contemporary Issues in Cybersecurity
}

\author{
Harry Katzan, Jr., Director, Institute for Cybersecurity Research, USA
}

\begin{abstract}
${ }^{1}$
The effectiveness of modern computer applications is normally regarded as a function of five basic attributes of secure computer and information systems: availability, accuracy, authenticity, confidentiality, and integrity. The concepts generally apply to government, business, education, and the ordinary lives of private individuals. The considerations normally involve extended Internet applications - hence the name Cybersecurity. Achieving and maintaining a secure cyberspace is a complicated process, and some of the concerns involve personal identity, privacy, intellectual property, the critical infrastructure, and the sustainability of organizations. The threats to a secure operating infrastructure are serious and profound: cyber terrorism, cyber war, cyber espionage, and cyber crime, to which the technical community has responded with safeguards and procedures, usually supplied by the private sector. This paper provides a comprehensive view of security in the cyber domain with the ultimate objective of developing a science of cybersecurity.
\end{abstract}

Keywords: Cybersecurity; Information Assurance; Critical Infrastructure Protection

\section{INTRODUCTION}

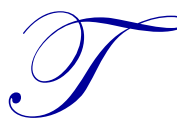

he Internet is the newest form of communication between organizations and people in modern society. Everyday commerce depends on it, and individuals use it for social interactions, as well as for reference and learning. To some, the Internet is a convenience for shopping, information retrieval, and entertainment. To others, such as large organizations, the Internet makes national and global expansion cost effective and allows disparate groups to profitably work together through reduced storage and communication costs. It gives government entities facilities for providing convenient service to constituents. The Internet is also efficient, because it usually can provide total service on a large variety of subjects in a few seconds, as compared to a much longer time for the same results that would have been required in earlier times (Katzan, 2012).

From a security perspective, the use of the term "cyber" generally means more than just the Internet, and usually refers to the use of electronics to communicate between entities. The subject of cyber includes the Internet as the major data transportation element, but can also include wireless, fixed hard wires, and electromagnetic transference via satellites and other devices. Cyber elements incorporate networks, electrical and mechanical devices, individual computers, and a variety of smart devices, such as phones, tablets, pads, and electronic game and entertainment systems. The near future portends road vehicles that communicate and driverless automobiles. A reasonable view would be that cyber is the seamless fabric of the modern information technology infrastructure that enables organizations and private citizens to sustain most aspects of modern everyday life.

Cyber supports the commercial, educational, governmental, and critical national infrastructure. Cyber facilities are pervasive and extend beyond national borders. As such, individuals, organizations, and nation-states can use cyber for productive and also destructive purposes. A single individual or a small group can use cyber for commercial gain or surreptitious invasion of assets. Activities in the latter category are usually classed as penetration and include attempts designed to compromise systems that contain vital information. In a similar vein, intrusion can also effect the day-to-day operation of critical resources, such as private utility companies.

Interconnectivity between elements is desirable and usually cost effective, so that a wide variety of dependencies have evolved in normal circumstances, and cyber intrusions have emerged. Thus, a small group of individuals can compromise a large organization or facility, which is commonly known as an asymmetric threat against which methodological protection is necessary. In many cases, a single computer with software obtained over the Internet

\footnotetext{
${ }^{1}$ This article is a partial reprint from the Journal of Service Science, 5(2), 71-78.
} 
can do untold damage to a business, utility, governmental structure, or personal information. Willful invasion of the property of other entities is illegal, regardless of the purpose or intent. However, the openness of the Internet often makes it difficult to identify and apprehend cyber criminals - especially when the subject's illegal actives span international borders.

\section{CYBERSECURITY OPERATIONS}

It is well established that cybersecurity is a complicated and complex subject encompassing computer security, information assurance, comprehensive infrastructure protection, commercial integrity, and ubiquitous personal interactions. Most people look at the subject from a personal perspective. Is my computer and information secure from outside interference? Is the operation of my online business vulnerable to outside threats? Will I get the item I ordered? Are my utilities safe from international intrusion? Have I done enough to protect my personal privacy? Are my bank accounts and credit cards safe? How do we protect our websites and online information systems from hackers? Can my identity be stolen? The list of everyday concerns that people have over the modern system of communication could go on and on. Clearly, concerned citizens and organizations look to someone or something else, such as their Internet service provider or their company or the government, to solve the problem and just tell them what to do.

So far, it hasn't been that simple and probably never will be. The digital infrastructure based on the Internet that we call cyberspace is something that we depend on every day for a prosperous economy, a strong military, and an enlightened lifestyle. Cyberspace, as a concept, is a virtual world synthesized from computer hardware and software, desktops and laptops, tablets and cell phones, and broadband and wireless signals that power our schools, businesses, hospitals, government, utilities, and personal lives through a sophisticated set of communication systems, available worldwide. However, the power to build also provides the power to disrupt and destroy. Many persons associate cybersecurity with cyber crime, since it costs persons, commercial organizations, and governments more than a $\$ 1$ trillion per year, (Obama, 2009). However, there is considerably more to cybersecurity than cyber crime, so it is necessary to start off with a few concepts and definitions.

Cyberspace has been defined as the interdependent network of information technology infrastructure, and includes the Internet, telecommunication networks, computer systems, and embedded processors and controllers in critical industries, (The White House, 2008). Alternately, cyberspace is often regarded as any process, program, or protocol relating to the use of the Internet for data processing transmission or use in telecommunication. As such, cyberspace is instrumental in sustaining the everyday activities of millions of people and thousands of organizations worldwide. The key terminology is that in a security event, a subject executes the crime against an object and that both entities incorporate computer and networking facilities.

\section{CYBER ATTACKS}

Cyber attacks can be divided into four distinct groups (Shackelford, 2012): cyber terrorism, cyber war, cyber crime, and cyber espionage. It would seem that cyber crime and cyber espionage are the most pressing issues, but the others are just offstage. Here are some definitions (Lord \& Sharp, 2011):

Cyber crime is the use of computers or related systems to steal or compromise confidential information for criminal purposes, most often for financial gain.

Cyber espionage is the use of computers or related systems to collect intelligence or enable certain operations, whether in cyberspace or the real world.

Cyber terrorism is the use of computers or related systems to create fear or panic in a society and may result in physical destruction by cyber agitation.

Cyber war consists of military operations conducted within cyberspace to deny an adversary, whether a state or nonstate actor, the effective use of information systems and weapons, or systems controlled by information technology, in order to achieve a political end. 
As such, cybersecurity has been identified as one of the most serious economic and national security challenges facing the nation (The White House, n.d). There is also a personal component to cybersecurity. The necessity of having to protect one's identity and private information from outside intrusion is a nuisance resulting in the use of costly and inconvenient safeguards.

\section{CYBERSPACE DOMAIN, ITS ELEMENTS AND ACTORS}

Cyberspace is a unique domain that is operationally distinct from the other operational domains of land, sea, air, and space. It provides, through the Internet, the capability to create, transmit, manipulate, and use digital information (McConnell, 2011). The digital information includes data, voice, video, and graphics transmitted over wired and wireless facilities between a wide range of devices that include computers, tablets, smart phones, and control systems. The Internet serves as the transport mechanism for cyberspace. The extensive variety of content is attractive to hackers, criminal elements, and nation states with the objective of disrupting commercial, military, and social activities. Below is a list of areas at risk in the cyberspace domain (Stewart, 2009). Many cyber events, classified as cyber attacks, are not deliberate and result from everyday mistakes and poor training. Others result from disgruntled employees. Unfortunately, security metrics include non-serious as well as serious intrusions, so that the cybersecurity threat appears to be overstated in some instances. This phenomenon requires that we concentrate on deliberate software attacks and how they are in fact related, since the object is to develop a conceptual model of the relationship between security countermeasures and vulnerabilities.

Areas at Risk in the Cyberspace Domain:

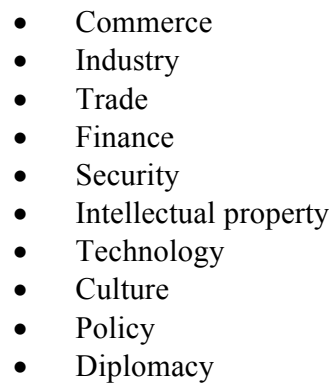

Many of the software threats can be perpetrated by individuals or small groups against major organizations and nation-states - referred to as asymmetric attacks, as mentioned previously. The threats are reasonably well known and are summarized below. It's clear that effective countermeasures are both technical and procedural, in some instances, and must be linked to hardware and software resources on the defensive side. The security risks that involve computers and auxiliary equipment target low-end firmware or embedded software, such as BIOS, USB devices, cell phones and tablets, and removable and network storage. Operating system risks encompass service packs, hotfixes, patches, and various configuration elements. Established counter measures, include intrusion detection and handling systems, hardware and software firewalls, and antivirus and anti-spam software.

Security Threats:

- Privilege escalation

- Virus

- Worm

- Trojan horse

- Spyware

- Spam

- Hoax

- Adware

- Rootkit

- Botnet

- Logic bomb 
The cybersecurity network infrastructure involves unique security threats and countermeasures. Most of the threats relate to the use of out-of-date network protocols, specific hacker techniques, such as packet sniffing, spoofing, phishing and spear phishing, man-in-the-middle attacks, denial-of-service procedures, and exploiting vulnerabilities related to domain name systems. Countermeasures include hardware, software, and protective procedures of various kinds. Hardware, software, and organizational resources customarily execute the security measures. There is much more to security threats and countermeasures, and the information presented here gives only a flavor to the subject.

There is an additional category of threats and countermeasures that primarily involves end-users and what they are permitted to do. In order for a threat agent to infiltrate a system, three elements are required: network presence, access control, and authorization. This subject is normally covered as the major features of information assurance and refers to the process of "getting on the system," such as the Internet or a local-area network. A threat agent cannot address a system if the computer is not turned on or a network presence is not possible. Once an end user is connected to the computer system or network, then access control and authorization take over. It has been estimated that $80 \%$ of security violations originate at the end-user level (Stewart, 2009). Access control concerns the identification of the entity requesting accessibility and whether that entity is permitted to use the system. Authorization refers to precisely what that entity is permitted to do, once permitted access. There is a high-degree of specificity to access-control and authorization procedures. For example, access control can be based on something the requestor knows, a physical artifact, or a biometric attribute. Similarly, authorization can be based on role, group membership, level in the organization, and so forth. Clearly, this category reflects considerations which the organizations has control over, and as such, constitutes security measures that are self-postulated.

\section{CYBERSECURITY COLLABORATION}

A collaboration group exists when a set of service providers $\boldsymbol{P}$ supplies a totality of services for a specific

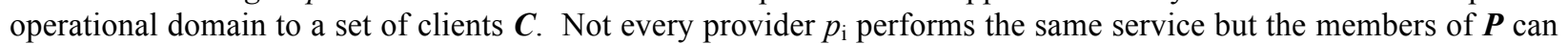
collectively supply all of the service needed for that domain. The client set $\boldsymbol{C}$ constitutes the functions in the operational system that require protection.

The controls that constitute a cyber security domain form a collaboration group. Diverse elements of hardware and software are used for network and operating system security. Clearly, processes are necessary for gaining network presence, access control to a given resource, and user authentication. Intrusion detection and prevention systems (IPDS) are implemented to perform continuous monitoring and cyber protection. Access roles and operational rules are developed to facilitate use of cyber security procedures and elements.

When a client adopts cybersecurity principles for network presence, access control, and authentication, for example, it applies the inherent methods for and by itself, thereby assuming the dual role of provider and client. Similarly, when an organization installs a hardware or software firewall for network protection, it is effectively applying a product for its own security.

In a security system, security controls exchange information and behavior in order to achieve mutually beneficial results. As security systems become more complex, the security entities adapt to optimize their behavior - a process often referred to as evolution (Mainzer, 1997). Differing forms of organization emerge such that the system exhibits intelligent behavior based on information interchange and the following nine properties: emergence, co-evolution, sub-optimal, requisite variety, connectivity, simple rules, self organization, edge of chaos, and nestability. Systems of this type are usually known as complex adaptive systems (Katzan, 2008). Complex adaptive systems are often known as "smart systems," and cybersecurity researchers are looking at the operation of such systems as a model for the design of cybersecurity systems that can prevent attacks through the exchange of information between security elements.

\section{DISTRIBUTED SECURITY}

The major characteristic of a cybersecurity system designed to prevent and mediate a cyber attack is that the totality of security elements in a particular domain are organized into a smart service system. This characteristic refers to the facility of cyber elements to communicate on a real-time basis in response to cyber threats. Currently, threat 
determination is largely manual and human-oriented. An intrusion detection system recognizes an intrusion and informs a security manager. That manager then contacts other managers via email, personal contact, or telephone to warn of the cyber threat. In a smart cybersecurity system, the intrusion detection software would isolate the cyber threat and automatically contact other elements in the domain to defend their system. Thus, the security service would handle intruders in a manner similar to the way biological systems handle analogous invasions: recognize the threat; attempt to neutralize it; and alert other similar elements.

In a definitive white paper on distributed security, McConnell (2011) recognizes the need for cyber devices to work together in near real-time to minimize cyber attacks and defend against them. This is a form of continuous monitoring and referred to as a cyber ecosystem in which relevant participants interact to provide security and maintain a persistent state of security. Clearly, a cyber ecosystem would establish a basis for cybersecurity through individually designed hierarchies of security elements, referred to as security devices. Ostensibly, security devices would be programmed to communicate in the event of a cyber attack. The conceptual building blocks of an ecosystem are automation, interoperability, and authentication. Automation refers to the notion of security devices being able to detect intrusion detection and respond to other security devices without human intervention. Thus, the security ecosystem could behave as a security service and provide speed and in the activation of automated prevention systems. Interoperability refers to the ability of the cyber ecosystem to incorporate differing assessments, hardware facilities, and organizations with strategically distinct policy structures. Authentication refers to the capability to extend the ecosystem across differing network technologies, devices, organizations, and participants.

Thus, the cyber ecosystem responds as a service system in requests for security service to participants that are members of the ecosystem, namely private firms, non-profit organizations, governments, individuals, processes, cyber devices comprised of computers, software, and communications equipment.

\section{MONROE DOCTRINE FOR CYBERSECURITY}

Internet governance refers to an attempt at the global level to legislate operations in cyberspace taking into consideration the economic, cultural, developmental, legal, political, and cultural interests of its stakeholders (Conway, 2007). A more specific definition would be the development and application by governments and the private sector of shared principles, norms, rules, decision-making, and programs that determine the evolution and use of the Internet (Conway, 2007). Internet governance is a difficult process because it encompasses, web sites, Internet service providers, hackers, and activists, involving differing forms of content and operational intent ranging from pornography and terrorist information to intrusion and malicious content. Cybersecurity is a complex form of service that purports to protect against intrusion, invasion, and other forms of cyber terrorism, crime, espionage, and war. But, attacks can be carried out by anyone with an Internet connection and a little bit of knowledge of hacking techniques. NATO has addressed the subject of cyber defense with articles that state the members will consult together in the event of cyber attacks but are not duty bound to render aid (Cavelty, 2011). It would seem that deterrence, where one party is able to suggest to an adversary that it is capable and willing to use appropriate offensive measures, is perhaps a useful adjunct to cybersecurity service. However, successful attribution of cyber attacks is not a fail proof endeavor so that offensive behavior is not a total solution to the problem of deterrence.

Cybersecurity is a pervasive problem that deserves different approaches. Davidson (2009) has noted an interesting possibility, based on the volume of recent cyber attacks. The context is that we are in a cyber war and a war is not won on strictly defensive behavior. A "Monroe Doctrine in Cyberspace" is proposed, similar to the Monroe Doctrine of 1823 that states "here is our turf; stay out or face the consequences."

\section{SUMMARY}

The Internet is a seamless means of communication between organizations and people in modern society; it supports an infrastructure that permits cost effective commerce, social interaction, reference, and learning. The use of the term "cyber" means more than just the Internet and refers to the use of electronics in a wide variety of forms between disparate entities. Cyber facilities are pervasive and extend beyond national borders and can be used by individuals, organizations, and nation states for productive and destructive purposes. A single individual or small 
group can use cyber technology for surreptitious invasion of assets to obtain vital information or to cause the disruption of critical resources.

Cybersecurity is conceptualized as a unique kind of service in which providers and clients collaborate to supply service through shared responsibility, referred to as collaborative security. Cybersecurity is achieved through distributed security implemented as a smart system with three important attributes: automation, interoperability, and authentication. A Monroe Doctrine for Cybersecurity is proposed.

\section{AUTHOR INFORMATION}

Professor Harry Katzan, Jr. is the author of books and papers on computer science, service science, and security. He teaches cybersecurity in the graduate program at Webster University and directs the Institute for Cybersecurity Research. His email address is katzanh@twc.com.

\section{REFERENCES}

Cavelty, M., (2011) Cyber-Allies: Strengths and Weaknesses of NATO's Cyberdefense Posture, IP - Global Edition, ETH Zurich.

Conway, M. (2007). Terrorism and Internet Governance: Core Issues, Dublin: Disarmament Forum 3.

Davidson, M. (2009, March 10). The Monroe Doctrine in Cyberspace, Testimony given to the Homeland Security Subcommittee on Emerging Threats, Cybersecurity, and Technology.

Katzan, H., (2008). Foundations of Service Science: A Pragmatic Approach, New York: iUniverse, Inc.

Katzan, H., (2008). Service Science: Concepts, Technology, Management, New York: iUniverse, Inc.

Katzan, H., (2010, January 4-6). Service Analysis and Design, International Applied Business Research Conference, Orlando, FL.

Katzan, H., (2010, January 4-6). Service Collectivism, Collaboration, and Duality Theory, International Applied Business Research Conference, Orlando, FL.

Katzan, H., (2012, October 4-5). Essentials of Cybersecurity, Southeastern INFORMS Conference, Myrtle Beach, SC.

Lord, K.M. and Sharp, T. (2011). America's Cyber Future: Security and Prosperity in the Information Age, 1, Center for New American Security.

Mainzer, K. (1997). Thinking in Complexity: The Complex Dynamics of Matter, Mind, and Mankind, New York: Springer.

McConnell, B. (2011, March 3). The Department of Homeland Security, Enabling Distributed Security in Cyberspace: Building a Healthy and Resilient Cyber Ecosystem with Automated Collective Action, Retrieved from http://www.dhs.gov/xlibrary/assets/nppd-cyber-ecosystem-white-paper-03-23-2011.pdf

Norman, D. (2011). Living with Complexity, Cambridge: The MIT Press.

Obama, B. H. (2009, May 29). Remarks by the U.S. President on Securing Our Nation's Cyber Infrastructure. The White House. East Room, Retrieved from https://www.whitehouse.gov/the-press-office/remarks-president-securing-our-nationscyber-infrastructure

Shackelford, S.L., (2012) In Search of Cyber Peace: A Response to the Cybersecurity Act of 2012, Stanford Law Review. Retrieved from http://www.stanfordlawreview.org/sites/default/files/online/articles/64-SLRO-106.pdf.

Stewart, J., (2009). CompTIA Security+ Review Guide, Indianapolis: Wiley Publishing, Inc.

The Department of Homeland Security (2009), National Infrastructure Protection Plan: Partnering to enhance protection and resiliency.

The Department of Homeland Security, More About the Office of Infrastructure Protection, (http://www.dhs.gov/xabout/structure/gc_1189775491423.shtm).

The White House, (2003, February). The National Strategy to Secure Cyberspace.

The White House, (2008, January 8). National Security Presidential Directive 54/Homeland Security Presidential Directive 23 (NSPD-54/HSPD-23).

The White House. (n.d) National Security Council, The Comprehensive National Cybersecurity Initiative, Retrieved from http://www.whitehouse.gov/cybersecurity/comprehensive-national-cybersecurity-initiative).

Vargo, S. and Akaka, M., (2009), Service-Dominant Logic as a Foundation for Service Science: Clarification, Service Science 1(1): $32-41$.

Working Group on Internet Governance, (2005 August) Report Document WSIS-II/PC-3/DOC/5-E. 